\title{
Luxación periastragalina medial pura: reporte de casos y revisión bibliográfica
}

\author{
Marcos R. Latorre, Javier E. Sánchez Saba, Mariano O. Abrego, Fernando Díaz Dilernia, Franco L. De Cicco, \\ Facundo Bilbao \\ Servicio de Ortopedia y Traumatología, Hospital Italiano de Buenos Aires, Ciudad Autónoma de Buenos Aires, Argentina
}

\begin{abstract}
RESUMEN
Las luxaciones subastragalinas (periastragalinas) sin fractura asociada son un cuadro poco frecuente. Representan solo el $1 \%$ de las luxaciones en el ser humano. Presentamos tres casos de luxaciones mediales puras en pacientes que sufrieron un traumatismo de alta energía. En todos los casos, el tratamiento consistió en la reducción cerrada bajo anestesia y posterior inmovilización; la evolución fue satisfactoria. Las luxaciones subastragalinas requieren de un diagnóstico temprano y una rápida resolución. Con estos casos se quiere demostrar la importancia de la sospecha diagnóstica y el beneficio de un tratamiento precoz y acertado. Palabras clave: Luxación periastragalina; complicaciones; reducción cerrada.

Nivel de Evidencia: IV
\end{abstract}

\section{Pure Medial Subtalar Dislocation: Case Report and Literature Review}

\begin{abstract}
Isolated subtalar dislocations without associated fracture are a rare entity. They represent only $1 \%$ of dislocations in humans. We present a series of 3 cases of pure medial dislocations in patients who suffered high-energy trauma. In all cases, the treatment was closed reduction under anesthesia and subsequent immobilization; all with good results. Subtalar dislocations require early diagnosis and rapid resolution. The aim of this presentation is to demonstrate the importance of diagnostic suspicion and the benefit of early treatment for successful outcome.
\end{abstract}

Key words: Subtalar dislocation; complications; closed reduction.

Level of Evidence: IV

\section{INTRODUCCIÓN}

Las luxaciones subastragalinas suelen asociarse a fracturas de astrágalo, maleolares e incluso metatarsianas. ${ }^{1}$ Las luxaciones puras son infrecuentes, en parte, debido a la presencia de estructuras ligamentarias fuertes y bien adaptadas para soportar la carga, como el ligamento astrágalo-calcáneo y el ligamento cervical. La principal causa de estas lesiones son los traumas de alta energía. ${ }^{2}$ El mecanismo de acción consiste en la inversión forzada del antepié cuando el pie está en flexión plantar.

Malgaigne y cols. ${ }^{3}$ describieron cuatro variantes: lateral, anterior, posterior y medial, esta última es la más frecuente. Las luxaciones mediales representan hasta el $85 \%$ de los casos. ${ }^{4}$ Tanto la articulación astrágalo-calcánea como la astrágalo-escafoidea están comprometidas. No obstante, las articulaciones astrágalo-cuboidea y astrágalotibial no suelen estar afectadas. ${ }^{5}$

Nuestro objetivo es comunicar tres casos de luxación periastragalina pura (sin fractura asociada) y realizar una revisión bibliográfica sobre el tema.

\footnotetext{
Recibido el 12-4-2020. Aceptado luego de la evaluación el 22-6-2020 • Dr. MARCOS R. LATORRE • marcos.latorre@ hospitalitaliano.org.ar Cómo citar este artículo: Latorre MR, Sánchez Saba JE, Abrego MO, Díaz Dilernia F, De Cicco FL, Bilbao F. Luxación periastragalina medial pura: reporte de casos y revisión bibliográfica. Rev Asoc Argent Ortop Traumatol 2021;86(1):83-90. https://doi.org/10.15417/issn.1852-7434.2021.86.1.1099
} 


\section{CASO CLÍNICO 1}

Mujer de 69 años, sin antecedentes de relevancia, acudió a la central de emergencias tras sufrir un trauma de alta energía al ser embestida por un automóvil. Tenía dolor asociado a impotencia funcional de pie y tobillo izquierdos. En primera instancia, había sido atendida en otro centro asistencial donde le realizaron una inmovilización con bota corta de yeso para el manejo del dolor, sin un diagnóstico presuntivo concluyente. Dentro de las $24 \mathrm{~h}$ del traumatismo, acudió a nuestra institución, porque persistía el dolor. Fue atendida de urgencia en la central de emergencias. Como primera medida se retiró la inmovilización para explorar la piel y las partes blandas. La paciente sufría intenso dolor en el pie y el tobillo izquierdos asociado a edema y equimosis (Figura 1).

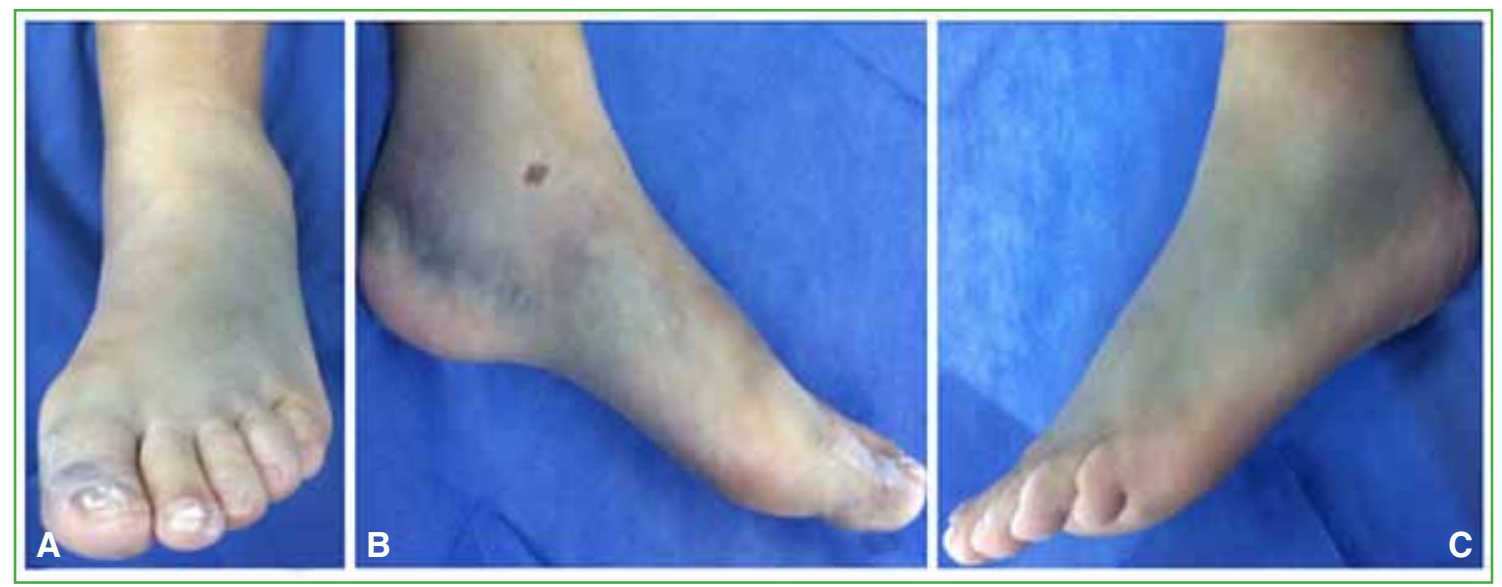

Figura 1. Imágenes clínicas que muestran el compromiso de partes blandas y el pie en flexión plantar con leve supinación. A. Frente. B. Cara medial. C. Cara lateral.

Las radiografías revelaron una luxación periastragalina medial, con evidente incongruencia de las articulaciones astrágalo-escafoidea y astrágalo-calcánea (Figura 2). La tomografía computarizada no mostró lesiones óseas asociadas (Figura 3).

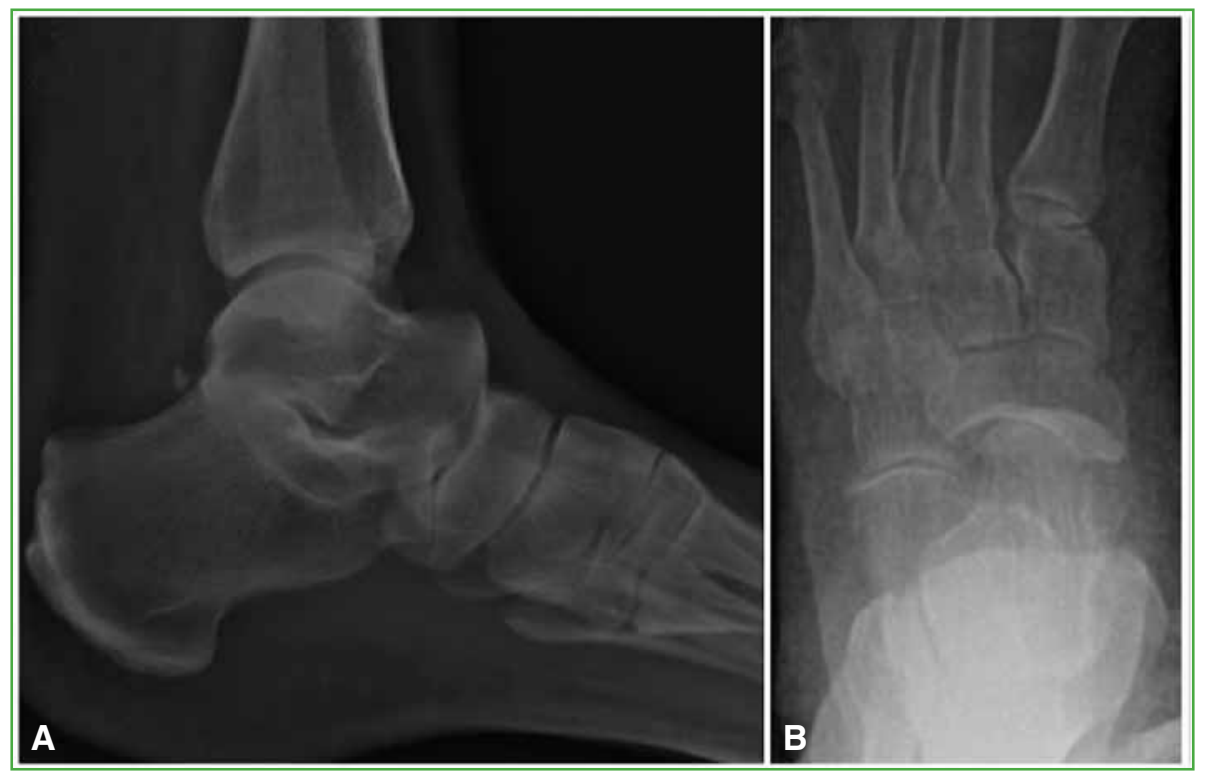

Figura 2. A. Radiografía de perfil que muestra la incongruencia en la articulación subastragalina y astrágalo-escafoidea. B. Radiografía de frente que revela una luxación periastragalina medial. 


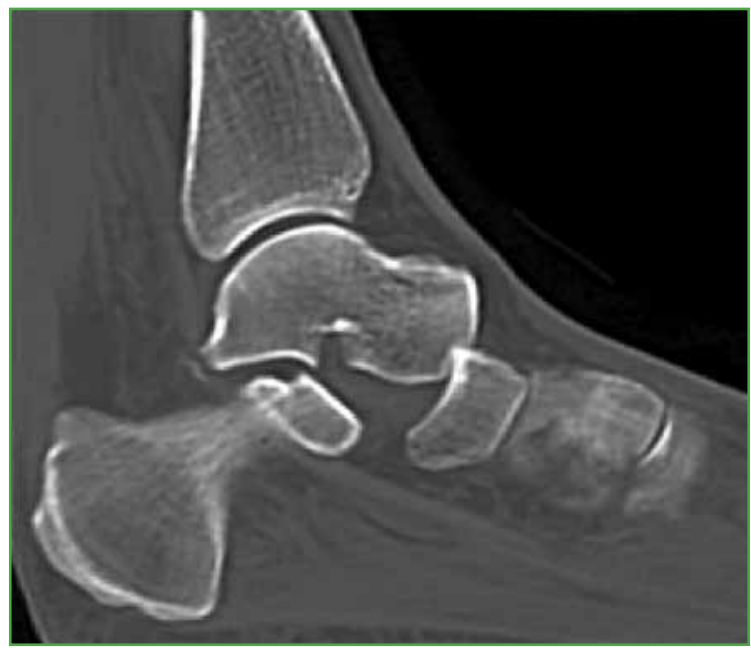

Figura 3. Tomografía computarizada. El borde superior del escafoides está desplazado hacia distal e impactado en la cara anterior del astrágalo.

Luxación transversal de la articulación astrágalo-calcánea.

Se decidió realizar la reducción cerrada urgente en el quirófano bajo anestesia general. Para relajar el tendón de Aquiles se efectuó la maniobra de flexión de rodilla. Se aplicó tracción en el eje, acentuando la deformidad, en primer término, para desimpactar y luego llevando el pie a la eversión, se consiguió la reducción. Se constató la adecuada reducción bajo radioscopia (Figura 4), se le colocó una bota corta de yeso, y se tomaron radiografías de control posoperatorias (Figura 5).

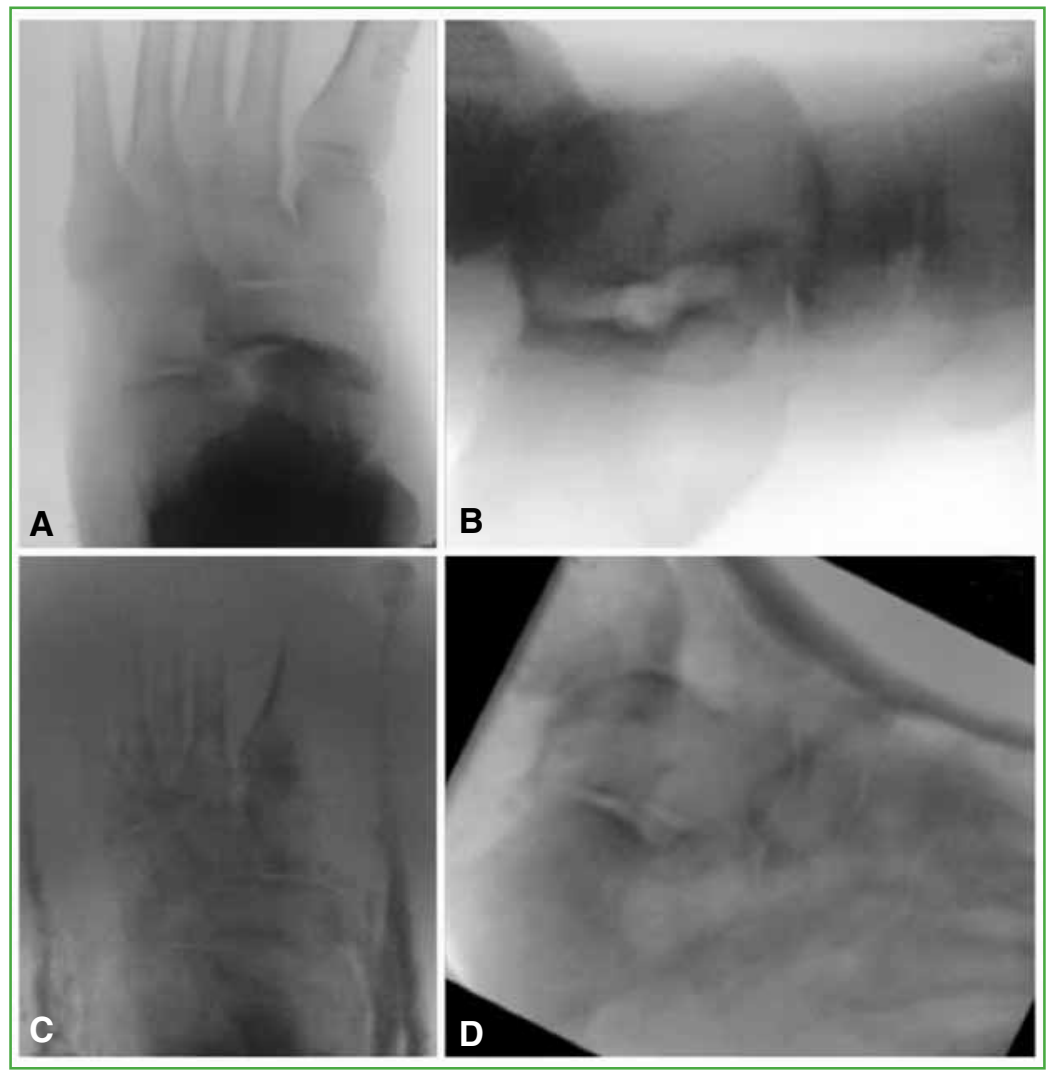

Figura 4. Radioscopia intraoperatoria. A y B. Antes de la reducción.

C y D. Después de la reducción. 


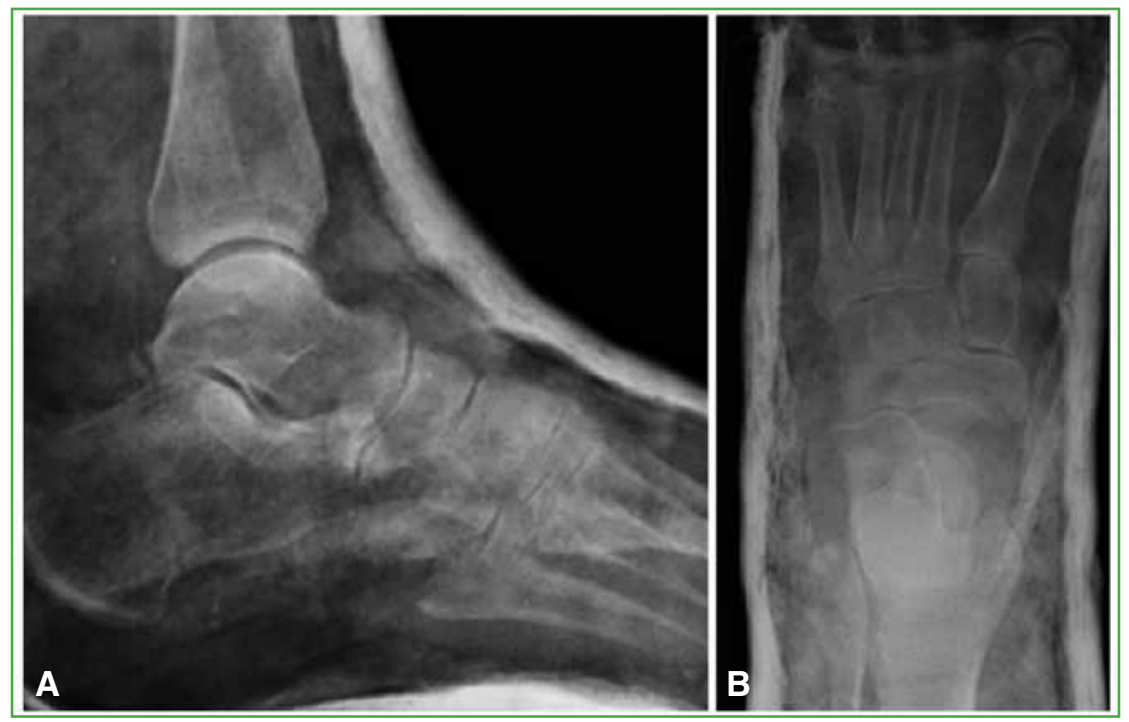

Figura 5. Control radiográfico posoperatorio. A. Perfil. B. Frente.

Se indicó inmovilización con yeso durante cuatro semanas. Luego se lo retiró, se colocó una bota walker y se permitió la carga parcial desde la semana 6. La paciente continuó con el protocolo de rehabilitación y se autorizó la carga completa sin restricciones desde el tercer mes. En el control a largo plazo, a los 12 meses, la paciente no tenía síntomas y, en el examen físico, se observó una adecuada estabilidad del retropié. El puntaje en la escala analógica visual era de $0 / 10$, el rango de movilidad era completo comparado con el contralateral y el puntaje de la AOFAS era de 100 (máximo 100).

\section{CASO CLÍNICO 2}

Hombre de 43 años que sufrió un traumatismo de tobillo derecho tras una caída desde un metro de altura. Consultó en la guardia de nuestro hospital el mismo día del traumatismo, tenía dolor, impotencia funcional y deformidad en dicho tobillo. Las radiografías mostraron una luxación periastragalina medial pura (Figura 6), por lo que fue sometido de urgencia a una reducción bajo anestesia (Figura 7).

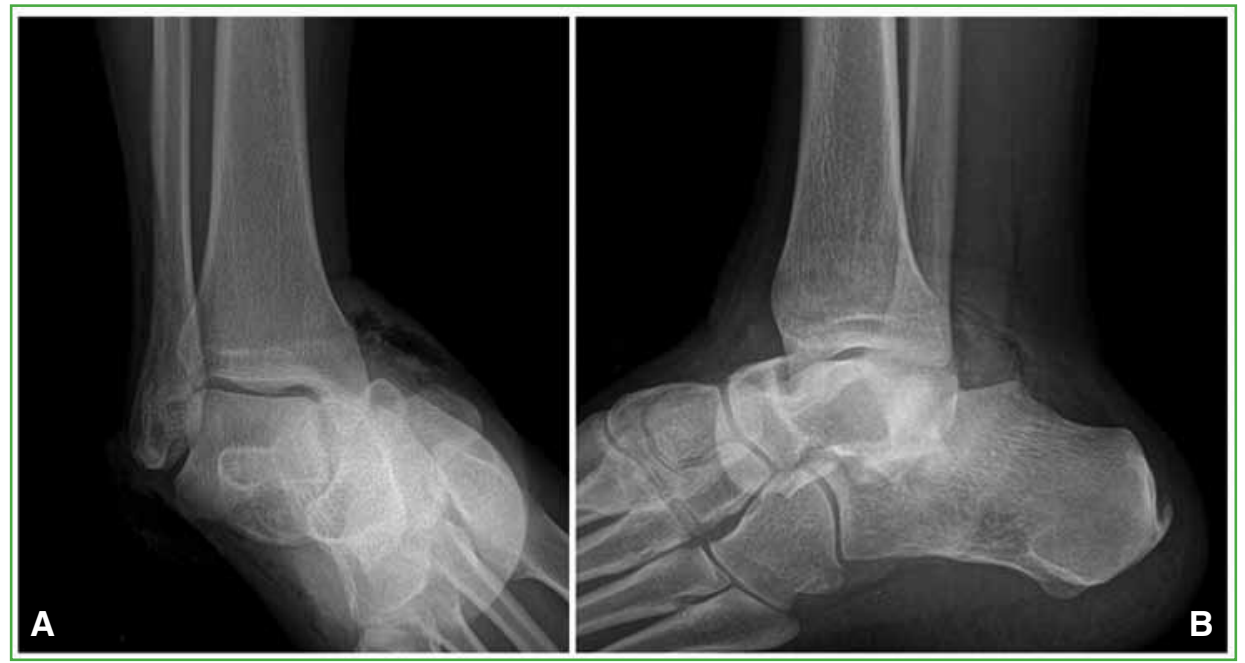

Figura 6. Radiografías de tobillo de frente (A) y de perfil (B). Luxación periastragalina medial. 


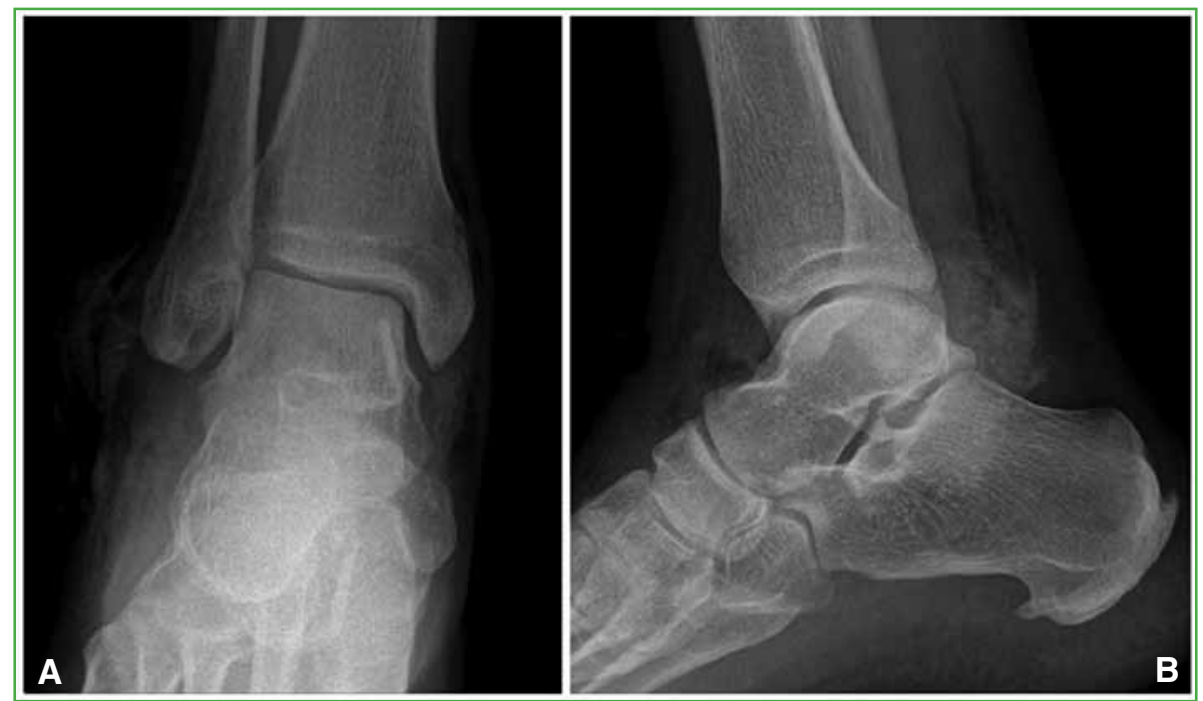

Figura 7. Control radiográfico después de la reducción. A. Frente. B. Perfil.

Se lo inmovilizó con bota corta de yeso. La inmovilización se mantuvo durante cuatro semanas para luego retirarla y colocar una bota walker por dos semanas más. A la semana 6, comenzó con carga parcial y progresó hacia la carga total a los tres meses. En el control anual, el puntaje en la escala analógica visual era de 2/10, con tobillo y retropié estables, el rango de movilidad era completo comparado con el contralateral, sentía molestias leves durante las actividades deportivas y el puntaje de la AOFAS era de 76 (máximo 100). Cabe destacar que el paciente presentó una fascitis plantar durante la evolución, que fue tratada con ejercicios de elongación asociados a terapia física y una infiltración (betametasona 10 mg más lidocaína al 2\%); los síntomas desaparecieron.

\section{CASO CLÍNICO 3}

Hombre de 44 años que sufrió un traumatismo de tobillo durante una actividad deportiva. Fue traído en ambulancia a nuestra central de emergencias, a las $6 \mathrm{~h}$ del traumatismo. Tenía dolor, deformidad e impotencia funcional del tobillo derecho. Las radiografías mostraron una incongruencia articular con luxación periastragalina medial (Figura 8).

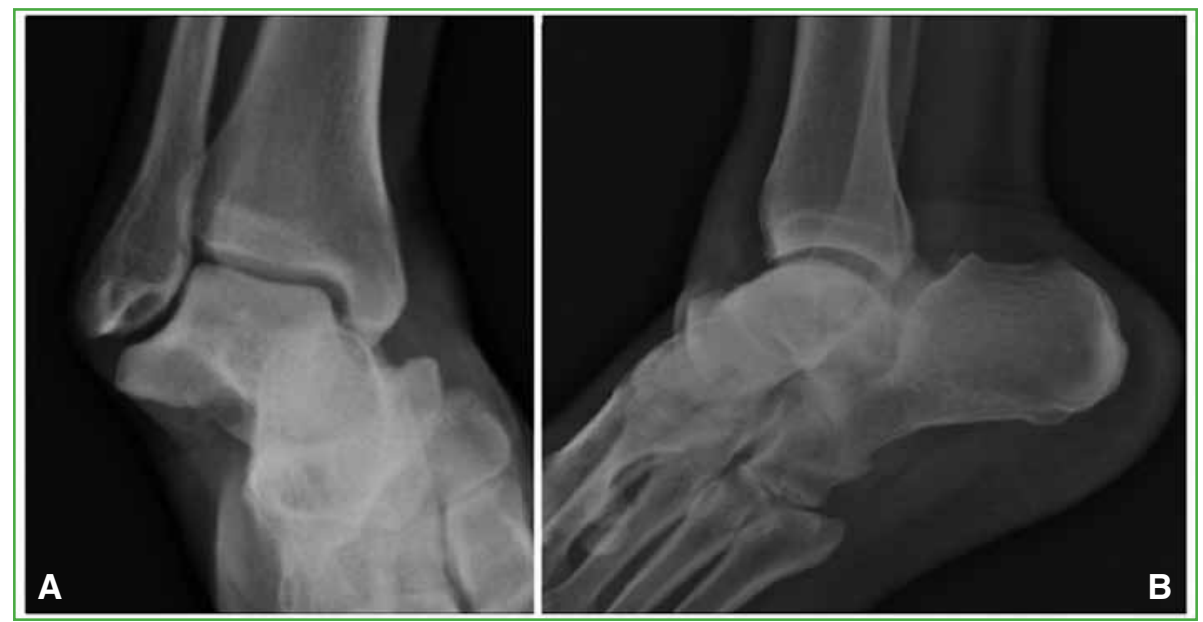

Figura 8. Radiografías de frente (A) y de perfil (B) que muestran la luxación medial. 
Se realizó la reducción cerrada de urgencia, bajo anestesia en el quirófano (Figura 9). Se lo inmovilizó con un yeso que se mantuvo durante cuatro semanas, luego usó una bota walker por dos semanas más y cumplió el protocolo de rehabilitación. Se autorizó la carga parcial desde la semana 6 y la carga total, a partir del tercer mes. El paciente interrumpió su seguimiento y consultó nuevamente luego de dos años de la cirugía, por lo cual no cumplió con el control anual estipulado. En el seguimiento alejado, el puntaje en la escala analógica visual fue de 0/10, no sentía molestias al practicar actividades deportivas. En el examen físico, se constató un rango de movilidad completo comparado con el contralateral y un puntaje de la AOFAS de 100 (máximo 100).

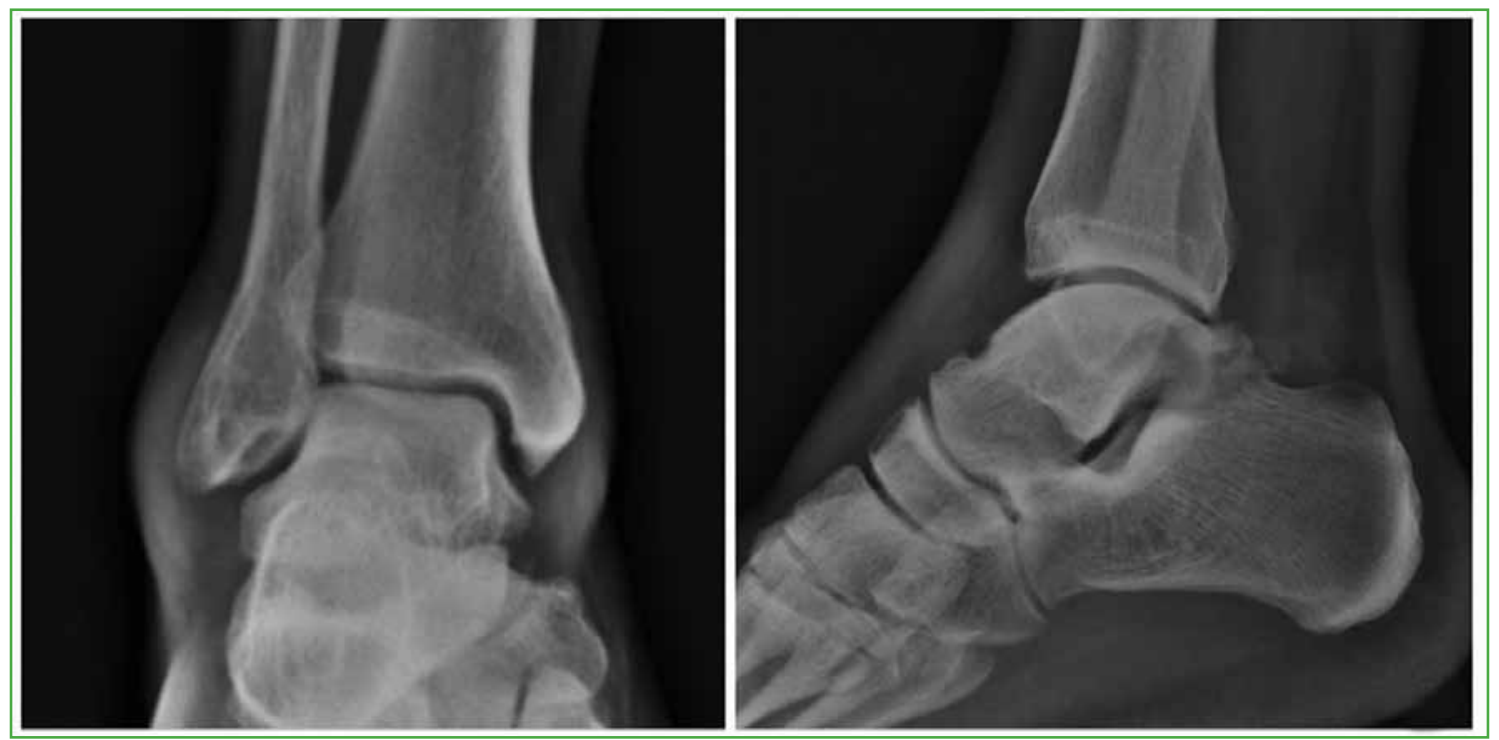

Figura 9. Radiografías de frente (A) y de perfil (B) que muestran una correcta reducción.

\section{DISCUSIÓN}

Las luxaciones periastragalinas son infrecuentes. Se caracterizan por la pérdida de las relaciones anatómicas normales entre el astrágalo, el calcáneo y el escafoides. Malgaigne y cols. clasificaron cuatro tipos de luxación; de ellas, la variante interna o medial es la más frecuente, con un rango estimado del $65-85 \% .^{3}$ Las luxaciones puras siguen siendo poco frecuentes. Algunos reportes analizan las lesiones del pie y tobillo asociadas a este tipo de luxaciones y comunican tasas que oscilan entre el $47 \%$ y el $88 \% .^{6-8}$

Este tipo de luxaciones son el producto de una inversión forzada del antepié cuando el pie está en flexión plantar. El sustentáculo del astrágalo actúa como fulcro. Si los ligamentos astrágalo-calcáneo y astrágalo-escafoideo se lesionan, el astrágalo permanece en su posición y se produce la luxación. ${ }^{9}$ En un estudio cadavérico, Ogiuchi y cols. hallaron que tanto el ligamento peroneo-calcáneo y el ligamento interóseo junto con la cápsula astrágaloescafoidea debían romperse para poder recrear una luxación medial (Figura 10). ${ }^{10}$

Inokuchi y cols. clasificaron las luxaciones mediales en dos grupos: las tipo "swift" y las tipo "shift". En las de tipo "swift", el calcáneo rota a medial, pero permanece por debajo del astrágalo, mientras que, en las de tipo "shift", el calcáneo se desvía a medial en relación con el astrágalo. ${ }^{11}$

En su serie de 25 casos, Bibbo y cols. comunicaron las complicaciones tempranas más frecuentes de las luxaciones subastragalinas: persistencia del dolor y limitaciones en el rango de movilidad. ${ }^{6} \mathrm{~A}$ su vez, utilizaron el puntaje de la AOFAS para tobillo y retropié, y hallaron diferencias significativas entre el miembro afectado (promedio 71) y el miembro contralateral (promedio 93). En nuestra serie, solo un paciente tuvo diferencias con respecto al pie y tobillo contralaterales (puntaje de la AOFAS 76/100), mientras que, en los otros dos casos, no se observaron diferencias con respecto al miembro no afectado. 


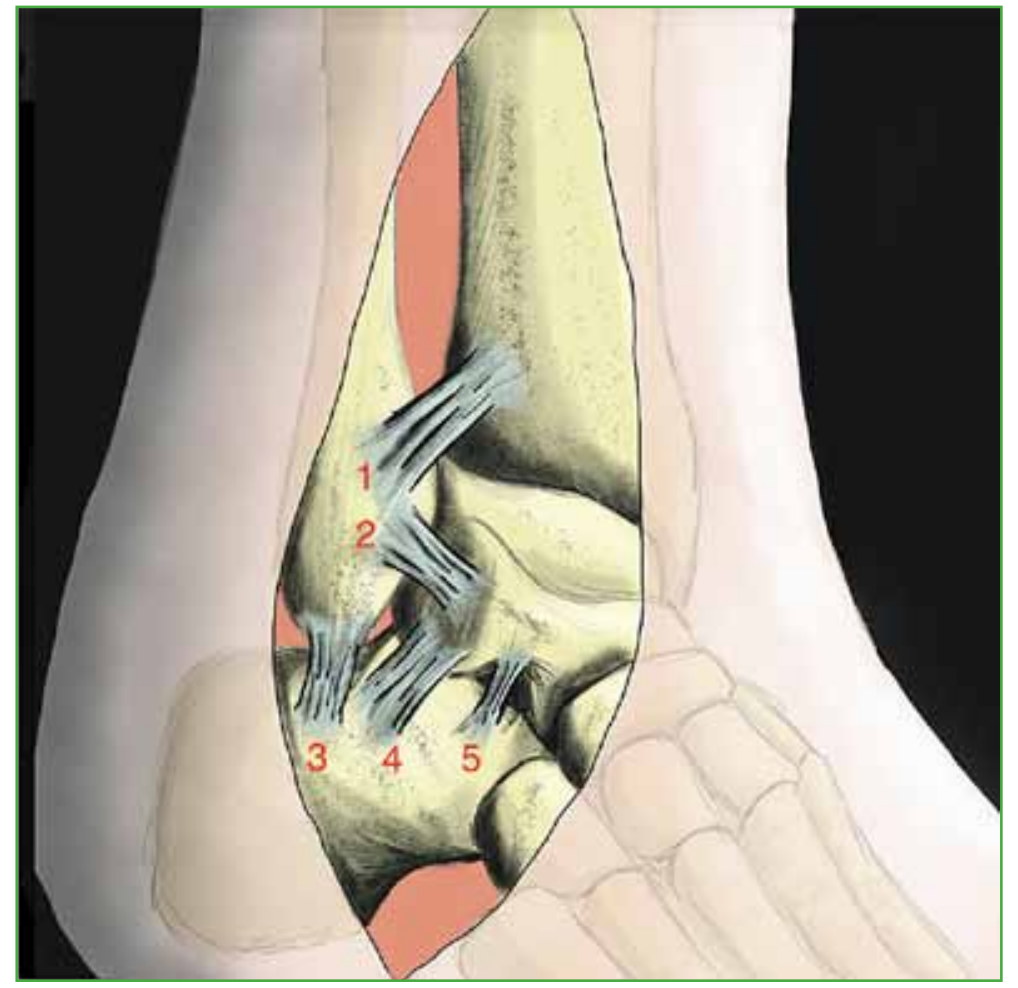

Figura 10. 1. Ligamento tibio-peroneo anterior, 2. ligamento peroneo-astragalino anterior, 3. ligamento peroneo-calcáneo, 4. ligamento astrágalo-calcáneo lateral, 5. ligamento interóseo.

Es frecuente observar artrosis postraumática de la articulación subastragalina, como una de las posibles complicaciones. Por otro lado, el dolor crónico después de la reducción no es tan común. ${ }^{7}$

Estudios previos establecen que el $10 \%$ de las luxaciones mediales son irreductibles mediante maniobras cerradas y requieren reducción abierta. ${ }^{12}$ La causa de la reducción dificultosa o fallida puede ser la interposición de distintos elementos, como el músculo extensor corto de los dedos, los tendones de los músculos peroneos largo y corto, y la cápsula de la articulación talo-navicular. El diagnóstico precoz, la reducción cerrada y la movilización temprana se asocian a un buen pronóstico. ${ }^{13}$ No obstante, las luxaciones laterales y aquellas que requieren reducción abierta suelen tener una mala evolución. ${ }^{14}$ Los tres pacientes presentados habían sufrido luxaciones periastragalinas mediales puras que fueron reducidas de urgencia, el intervalo entre el traumatismo y el procedimiento fue inferior a $12 \mathrm{~h}$, y luego fueron inmovilizadas.

\section{CONSIDERACIONES FINALES}

Si bien las luxaciones subastragalinas son cuadros poco frecuentes, no dejan de ser importantes debido a la magnitud de la lesión. Siempre se debe considerar la asociación de esta lesión con politraumatismos, accidentes en la vía pública y traumas de alta energía. La sospecha diagnóstica es fundamental, ya que el diagnóstico correcto de la lesión permite indicar un tratamiento oportuno y así evitar las complicaciones que puedan poner en riesgo la articulación subastragalina.

Con la presentación de estos tres casos se quiere demostrar la importancia de la sospecha diagnóstica y el beneficio de un tratamiento precoz y acertado para garantizar buenos resultados terapéuticos.

Conflicto de intereses: Los autores no declaran conflictos de intereses. 


\section{BIBLIOGRAFÍA}

1. Perugia D, Basile A, Massoni C, Gumina S, Rossi F, Ferretti A. Conservative treatment of subtalar dislocations. Int Orthop 2002;26:56-60. https://doi.org/10.1007/s002640100296

2. Tucker DJ, Burian G, Boylan JP. Lateral subtalar dislocation: review of the literature and case presentation. J Foot Ankle Surg 1998;37(3):239-47. https://doi.org/10.1016/s1067-2516(98)80118-x

3. Malgaigne JF, Burger DC. Die Knochenbrüche und VerrenKungen. Stuttgart, Germany: Rieger; 1856;(11):820.

4. Saltzman C, Marsh JL. Hindfoot dislocations: when are they not benign? J Am Acad Orthop Surg 1997;5(4):192198. https://doi.org/10.5435/00124635-199707000-00002

5. Freund KG. Subtalar dislocations: a review of the literature. J Foot Ankle Surg 1989;28(5):429-32. PMID: 2685089

6. Bibbo C, Anderson R, Davis W. Injury characteristics and the clinical outcome of subtalar dislocations: a clinical and radiographic analysis of 25 cases. Foot Ankle Int 2003;24(2):158-63. https://doi.org/10.1177/107110070302400210

7. DeLee JC, Curtis RC. Subtalar dislocation of the foot. J Bone Joint Surg Am 1983;64:433-7. PMID: 7061560

8. Merchan EC. Subtalar dislocations: long-term follow-up of 39 cases. Injury 1992;23:97-100. https://doi.org/10.1016/0020-1383(92)90041-p

9. Giannoulis D, Papadopoulos D, Lykissas M, Koulouvaris P, Gkiatas I, Mavrodontidis A. Subtalar dislocation without associated fractures: Case report and review of literature. World J Orthop 2015;6(3):374-9. https://doi.org/10.5312/wjo.v6.i3.374

10. Ogiuchi M, Suzuki K, Takahashi S. Experience of subtalar dislocation. JJTOM 1974;1172-5.

11. Inokuchi S, Hashimoto T, Usami N. Posterior subtalar dislocation. J Trauma 1997;42:310-13. https://doi.org/10.1016/S0958-2592(96)90016-4

12. Heckman JD. Fractures of the talus. En: Bucholz RW, Heckman JD (eds.). Rockwood and Green's fractures in adults, $5^{\mathrm{a}}$ ed. Philadelphia: Lippincott Williams and Wilkins; 2001:2091-132.

13. Merianos P, Papagiannakos K, Hatzis A, Tsafantakis E. Peritalar dislocation: a follow-up report of 21 cases. Injury 1988;19(6):439-42. https://doi.org/10.1016/0020-1383(88)90143-x

14. Garofalo R, Moretti B, Ortolano V, Cariola P, Solarino G, Wettstein M, et al. Peritalar dislocations: a retrospective study of 18 cases. J Foot Surg 2004;43(3):166-72. https://doi.org/10.1053/j.jfas.2004.03.008 